\title{
Effects of cyclic stretch on the molecular regulation of myocardin in rat aortic vascular smooth muscle cells
}

\author{
Chiung-Zuan Chiu ${ }^{1,2}$, Bao-Wei Wang ${ }^{1,2}$ and Kou-Gi Shyu $u^{2,3^{*}}$
}

\begin{abstract}
Background: The expression of myocardin, a cardiac-restricted gene, increases during environmental stress. How mechanical stretch affects the regulation of myocardin in vascular smooth muscle cells (VSMCs) is not fully understood. We identify the mechanisms and pathways through which mechanical stretch induces myocardin expression in VSMCs.

Results: Rat VSMCs grown on a flexible membrane base were stretched to $20 \%$ of maximum elongation, at 60 cycles per min. An in vivo model of aorta-caval shunt in adult rats was also used to investigate myocardin expression. Cyclic stretch significantly increased myocardin and angiotensin II (Angll) expression after 18 and $6 \mathrm{~h}$ of stretch. Addition of extracellular signal-regulated kinases (ERK) pathway inhibitor (PD98059), ERK small interfering RNA (siRNA), and Angll receptor blocker (ARB; losartan) before stretch inhibited the expression of myocardin protein. Gel shift assay showed that myocardin-DNA binding activity increased after stretch. PD98059, ERK siRNA and ARB abolished the binding activity induced by stretch. Stretch increased while myocardin-mutant plasmid, PD98059, and ARB abolished the promoter activity. Protein synthesis by measuring $\left[{ }^{3} \mathrm{H}\right]$ proline incorporation into the cells increased after cyclic stretch, which represented hypertrophic change of VSMCs. An in vivo model of aorta-caval shunt also demonstrated increased myocardin protein expression in the aorta. Confocal microscopy showed increased VSMC size $24 \mathrm{~h}$ after cyclic stretch and VSMC hypertrophy after creation of aorta-caval shunt for 3 days.
\end{abstract}

Conclusions: Cyclic stretch enhanced myocardin expression mediated by Angll through the ERK pathway in cultured rat VSMCs. These findings suggest that myocardin plays a role in stretch-induced VSMC hypertrophy.

Keywords: Myocardin, Stretch, Vascular smooth muscle cells, ERK pathway

\section{Background}

In recent years, vascular smooth muscle cell (VSMC) hypertrophy has been increasingly associated with the development of atherosclerotic disease [1]. Hypertrophy of VSMCs may result in plaque rupture and vulnerability in atherosclerosis [2]. Hypertrophy of VSMCs may be induced by certain cardiac-restricted genes through specific pathways under different types of environmental stress [3-5].

\footnotetext{
*Correspondence: shyukg@ms12.hinet.net

${ }^{2}$ Division of Cardiology, Shin Kong Wu Ho-Su Memorial Hospital, 95 Wen-

Chang Rd, Taipei, Taiwan

${ }^{3}$ Graduate Institute of Clinical Medicine, College of Medicine, Taipei Medical

University, Taipei, Taiwan

Full list of author information is available at the end of the article
}

Myocardin is a potent cardiovascular regulated gene and transcriptional cofactor, which functionally synergizes with serum response factor (SRF; a transcriptional factor) and has been documented to have measurable effects on cardiac embryo development and both VSMC and cardiomyocyte hypertrophy [6-10]. An earlier study showed myocardin knockout mice resulted in embryonic lethality at E10.5, and was associated with failed VSMC differentiation [7]. Previous studies performed on rat carotid artery VSMCs following vascular injury have demonstrated that myocardin can selectively regulate SRF binding to the degenerate CArGs on VSMC actin to increase transcriptional activity. Loss of myocardin may contribute to the supression of actin expression in response to vascular injury. Vascular injury has been associated with induced

\section{Biomed Central}


expression of myocardin [11]. In addition, transfection with dominant-negative forms and small interfering RNA (siRNA) of myocardin in cultured VSMC decreased transcription of VSMC maker genes. Previous reports also indicated that either exogenous addition or stress-induced expression of angiotensin II (AngII) secretions would result in myocardin expression and subsequent VSMC or cardiac myocyte hypertrophy [12-15]. So myocardin itself may act as a cardiac regulated gene and cooperate with AngII in regulating VSMC or/and cardiac myocyte hypertrophy through specific signal transduction pathways under conditions of stress or injury to the cardiovascular system.

The application of cyclic stretch to cultured VSMCs has been widely used as an in vitro experiment to study molecular events in response to mechanical overload [16-20]. It has previously been reported that cyclic mechanical stretch induced hypertrophy in VSMCs [21-24]. Cells in the cardiovascular system are permanently subjected to mechanical forces due to the pulsatile variation of blood flow and shear force, created by the beating heart. These hemodynamic forces play an important role in the regulation of vascular development, remodeling, repair and formation of atherosclerotic stenosis [25-28]. Mechanical stretch can modulate several different cellular functions in VSMCs. These functions may include cell proliferation and differentiation, migration, survival or apoptosis, vascular remodeling, as well as autocrine or paracrine functions $[29,30]$. This study aimed to identify the cellular and molecular effects of mechanical stretch on VSMCs regulated by myocardin, and to identify its signal transduction pathway and relationship with AngII. Knowing the impact of mechanical stretch on the cardiovascular system is crucial to the understanding of the pathogenesis of cardiovascular diseases, and a key to providing new insight into the prevention and therapy of cardiovascular diseases.

Previous reports have provided strong evidence that myocardin plays an important role in VSMC hypertrophy related to AngII secretion [12]. However, no previous study has shown how cyclic mechanical stretch affects myocardin in the hypertrophy of VSMCs. Thus, in this study, we firstly investigated the mechanism of myocardin expression in cyclic mechanical stretch. Secondly, we investigated the effect and signal transduction pathway of myocardin expression induced by cyclic stretch.

\section{Methods}

Vascular smooth muscle cell culture

Primary cultures of VSMC were grown by the explant technique from the thoracic aorta of 200-250 g male Sprague-Dawley rats, as previously described $[31,32]$. Cells were cultured in medium containing $20 \%$ fetal calf serum, $0.1 \mathrm{mmol} / \mathrm{L}$ non-essential amino acids, $1 \mathrm{mmol} / \mathrm{L}$ sodium pyruvate, $4 \mathrm{mmol} / \mathrm{L}$ L-glutamine, $100 \mathrm{U} / \mathrm{mL}$ penicillin, and $100 \mathrm{mg} / \mathrm{mL}$ streptomycin at $37^{\circ} \mathrm{C}$ under $5 \% \mathrm{CO} 2 / 95 \%$ air in a humidified incubator. When confluent, monolayers of VSMCs were passaged every 6-7 days after trypsinization and were used for experiment from the $4^{\text {th }}$ to $6^{\text {th }}$ passages. These $4^{\text {th }}$ to $6^{\text {th }}$ passage cells were then cultured in Flexcell I flexible membrane dishes in medium containing $0.5 \%$ fetal calf serum, and the cells were incubated for a further 2 days to render them quiescent before initiating each experiment. The study was reviewed and approved by the Institutional Animal Care and Use Committee of Shin Kong Wu Ho-Su Memorial Hospital and conforms to Guide for the Care and Use of Laboratory Animals published by the US National Institutes of Health (NIH Publication No. 85-23, revised 2011).

\section{In vitro cyclic stretch on cultured vascular smooth muscle cells}

The strain unit Flexcell FX-2000 (Flexcell International Co., NC, USA) consists of a vacuum unit linked to a valve controlled by a computer program. VSMCs cultured on the flexible membrane base were subjected to cyclic stretch produced by this computer-controlled application of sinusoidal negative pressure, as previously characterized and described in detail [33,34]. A $10 \%$ or $20 \%$ cyclic stretch was performed with a frequency of $1 \mathrm{~Hz}$ (60 cycles/min).

\section{Antibodies and reagents}

Rabbit polyclonal antibodies against myocardin, mouse monoclonal antibodies (mAbs) against c-Jun $\mathrm{N}$-terminal kinase (JNK) and anti-GAPDH antibodies were obtained from Santa Cruz Biotechnology (Santa Cruz, CA). Mouse mAbs against p38 mitogen-activated protein kinase (MAPK), extracellular signal-regulated kinase (ERK), and phospho-ERK were purchased from BD Bioscience Pharmingen (San Diego, CA). PD98059, SB203580, and SP600125 were purchased from Calbiochem (San Diego, $\mathrm{CA}$ ). All other chemicals of reagent grade were obtained from Sigma (St Louis, MO). The roles of JNK, p38 MAPK, and ERK in stretch-induced myocardin expression were determined by pretreatment of the VSMCs with $25 \mu \mathrm{M}$ SP600125, $3 \mu \mathrm{M}$ SB203580, or $50 \mu \mathrm{M}$ PD98059 for 30 min before cyclic stretch. SP600125 is a potent, cell-permeable, selective, and reversible inhibitor of JNK. SB203580 is a highly specific, cell-permeable inhibitor of p38 MAPK. PD98059 is a specific and potent inhibitor of the ERK pathway. The AngII and AngII antibodies came from Bachem AG (Torrance, CA). To examine the effect of ARB (AngII type 1 receptor blocker), VSMCs were treated with $100 \mathrm{nM}$ losartan (Merck \& Co, Inc). 


\section{Western blot analysis}

Western blot was performed as previously described [35].

\section{Real-time RT-PCR}

Total RNA was isolated from VSMCs using the singlestep acid guanidinium hiocyanate/phenol/chloroform extraction method. The cDNA produced by reverse transcription (RT) was used to generate myocardin probes by polymerase chain reaction (PCR) as previously described [17]. Primers were designed for detection of myocardin gene expression. The primers for myocardin were: 5 '-GG ACTGCTCTGGCAACCCAGTGC-3'; reverse: 5' -CATC TGCTGACTCCGGGTCATTTGC - 3'. GAPDH gene expression was used as internal controls. The primers for GAPDH were: 5'-GAGAGGCTCTCTGTCGACTAC-3'; reverse: 5' -TAGTGTAGGTTGGGCGCTCAA-3'.

\section{RNA interference}

VSMCs were transfected with $800 \mathrm{ng}$ of ERK- and myocardin-annealed siRNA (Dharmacon, Lafayette, CO). ERK siRNAs are target- specific 20- to 25-nt siRNAs designed to knock down gene expression. The siRNA sequences were 5'-GACCGGAUGUUAACCUUUAUU (sense) and 5'-PUAAAGGUUAACA UCCGGUCUU (antisense) for ERK. The myocardin siRNA sequences were 5'-UGCAACUGCAGAAGCAGAAUU (sense) and 5'-UGCAACUGGUCUUGCAGAAUU (antisense). As a negative control, a non-targeting (control) siRNA (Dharmacon) was used. For transfection of rat VSMCs with siRNA oligonucleotides, we used Effectene transfection reagent according to the manufacturer's instructions (Qiagen, Valencia, CA). After incubation at $37^{\circ} \mathrm{C}$, cells were subjected to stretch and analyzed by Western blot.

\section{Measurement of Angll concentration by enzyme-linked immunosorbent assay (ELISA)}

Conditioned medium from VSMCs subjected to cyclic stretch and those from unstretched cells were collected for AngII measurement. The level of AngII was measured by a quantitative sandwich enzyme immunoassay technique (R\&D Systems, Minneapolis, MN, USA). The lowest limit of AngII ELISA kit was $52 \mathrm{pg} / \mathrm{mL}$.

\section{Electrophoretic mobility shift assay (EMSA)}

Nuclear protein concentrations from cultured VSMCs were determined by the Bradford method as commercialized by Santa Cruz Biotechnology and EMSA was performed as previously described [13]. Consensus and control oligonucleotides were labeled by polynucleotide kinase incorporation of $\left[\gamma^{-}{ }^{32} \mathrm{P}\right]$ ATP. In each case, mutant or cold oligonucleotide was used as a control to compete with the labeled sequences. The oligonucleotide sequence of consensus binding site for SRF was sense: 5' - GGA
TGT CCA TAT TAG GAC ATC T-3' and reverse: $5^{\prime}-$ CCT ACA GGT ATA ATC CTG TAG A-3'. The mutant oligonucleotide sequence was 5' - GGA TGT CCA TAT TAT TAC ATC T-3'.

\section{Promoter activity assay}

A -968 to +44 bp rat myocardin promoter construct was generated as previously described [13]. The myocardin promoter contains myocardin binding sites for SRF (sequences: CGGTTTAGGG) located at -514 to $-505 \mathrm{bp}$ of the promoter region. We used the binding sites to detect the transcriptional activity of myocardin. For construction of the mutant SRF binding region of myocardin, we changed the sequences located at -507 to $-506 \mathrm{bp}$ from CGGTTTAGGG to CGGTTTATTG by using a mutagenesis kit (Stratagene, La Jolla, CA). Site-specific mutations were confirmed by DNA sequencing. Plasmids were transfected into VSMCs using a low-pressure accelerated gene gun (Bioware, Taipei, Taiwan). Rat genomic DNA was amplified with forward (GGACTGCTCTGGCAAC CCAGT GC) and reverse (CATCTGCTGACTCCGGG TCATTTGC) primers. The amplified product was digested with $M l u$ I and $B g l$ II restriction enzymes and ligated into pGL3-basic luciferase plasmid vector (Promega, Madison, WI) digested with the same enzymes. In brief, $2 \mu \mathrm{g}$ of plasmid DNA was suspended in $5 \mathrm{~mL}$ of PBS and was delivered to the cultured VSMCs at a helium pressure of 15 psi. The transfection efficiency using this method is $30 \%$. Following $12 \mathrm{~h}$ of cyclic stretching, cell extracts were prepared using the Dual-Luciferase Reporter Assay System (Promega) and measured for dual luciferase activity by luminometer (Turner Designs, Sunnyvale, CA, USA).

\section{Migration assays}

The migration activity of VSMCs was determined using the growth-factor-reduced Matrigel invasion system (Becton Dickinson), following the protocol provided by the manufacturer. The migration assay was performed as previously described [17].

\section{Determination of protein synthesis}

Protein synthesis was examined by measuring $\left[{ }^{3} \mathrm{H}\right]$ proline incorporation into the cells. Cultured VSMCs were divided into the following groups: (1) control group: the cells were cultured in serum-free DMEM; and (2) mechanical stretch group ( $20 \%$ cyclic stretch) added to serum-free medium. Each experiment was repeated 6 times. VSMCs were first grown in DMEM with 10\% FBS and $200 \mathrm{mg} / \mathrm{L} \mathrm{L}$-glutamine, and then seeded in 24-well plates at $1 \times 10^{5}$ cells/well in DMEM $+10 \%$ FBS. After synchronization of VSMCs, the medium was changed to DMEM without serum. VSMCs were treated with cyclic stretch and exposed to $\left[{ }^{3} \mathrm{H}\right]$ proline at the concentration of $1 \mu \mathrm{Ci} /$ well for the last $12 \mathrm{~h}$ of the $24 \mathrm{~h}$ incubation period. 
After the incubation, the cells were washed with ice-cold PBS and $10 \%$ trichloroacetic acid. Acid-insoluble $\left[{ }^{3} \mathrm{H}\right]$ proline was collected on glass fiber filters (Whatman, Kent, UK) and determined by a liquid scintillation counter (LS 6500, Beckman, Fullerton, CA , USA).

\section{Rat model of aorta-caval shunt}

The aorta-caval shunt was produced as previously described [17]. The vena cava and aorta were exposed via abdominal midline incision. In brief, the aorta was punctured at the union of the segment two-thirds caudal to the left renal artery and one-third cephalic to the aortic bifurcation, with an 18-gauge disposable needle held with a plastic syringe. The needle was advanced into the aorta, perforating its adjacent wall and penetrating the vena cava. The induced aorta-caval shunt produced a ratio of 1.7 of pulmonary to systemic flow. Sham-operated control animals were prepared in a similar manner, except that the aorta was not punctured.

\section{Statistical analysis}

All results were expressed as means \pm SEM. Statistical significance was evaluated using variance (GraphPad Software Inc., San Diego, CA, USA). Dunnett's test was used to compare multiple groups to a single control group. Tukey-Kramer comparison was used for pairwise comparisons between multiple groups after ANOVA. A value of $\mathrm{P}<0.05$ was considered to denote statistical significance.

\section{Results}

\section{Cyclic stretch enhances myocardin protein and mRNA} expression in vascular smooth muscle cells

The level of myocardin protein began to increase as early as $6 \mathrm{~h}$ after stretch to $20 \%$ elongation, reaching a maximum of 3.1-fold over the control by $18 \mathrm{~h}$, remaining elevated up to $24 \mathrm{~h}$ and tending to decline at $30 \mathrm{~h}$. When VSMCs were stretched at $10 \%$ elongation, the level of myocardin protein was similar to that of controls without stretch (Figure 1A and B). The real-time PCR showed that myocardin mRNA expression increased maximally after $18 \mathrm{~h}$ of stretch at 20\% elongation (Figure 1C). These results indicated that cyclic stretch induced myocardin expression in VSMCs.

\section{Stretch-induced myocardin protein expression in vascular smooth muscle cells is mediated by the ERK pathway}

To identify the possible signal pathway mediating the stretch-induced myocardin expression in VSMCs, the VSMCs were stretched $20 \%$ for $24 \mathrm{~h}$ in the presence and absence of inhibitors or siRNA. As shown in Figure 2, the stretch-induced increases of myocardin proteins were significantly blocked when PD98059 (ERK pathway inhibitor; $50 \mu \mathrm{M})$ and ERK siRNA were added $30 \mathrm{~min}$ before

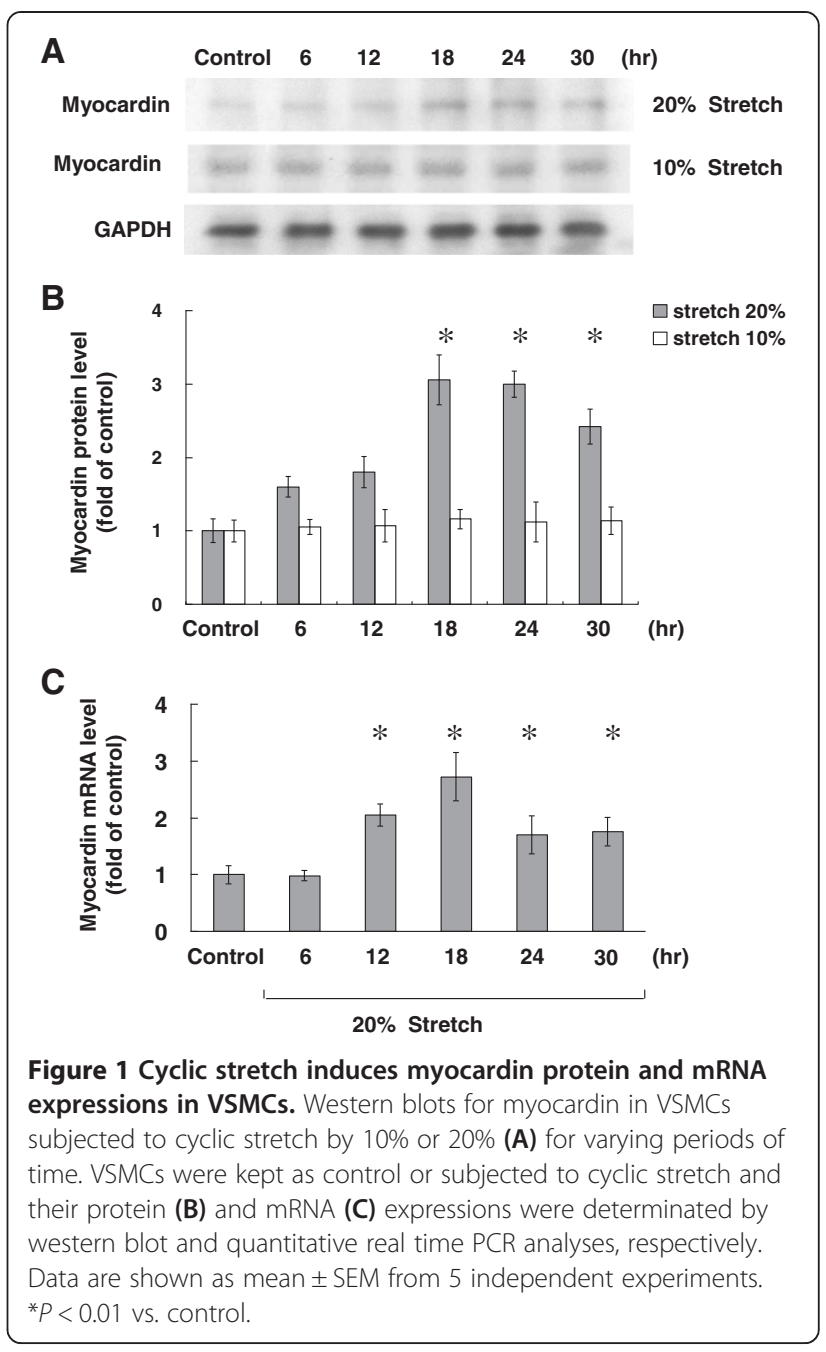

stretch. The myocardin proteins induced by stretch were not affected by the addition of SP600125 $(20 \mu \mathrm{M})$ or SB203580 $(30 \mu \mathrm{M})$. To test the specific effect of ERK MAPK (Mitogen-Activated Protein Kinase) pathway on mediating the expression of myocardin, ERK siRNA was transfected to VSMCs before cyclic stretch. Moreover, ERK siRNA also completely blocked the myocardin expression induced by stretch (Figure 2B). The DMSO (Dimethyl sulfoxide) alone as a vehicle control and control siRNA did not affect the myocardin expression induced by cyclic stretch. These findings implied that the ERK pathway, but not JNK or p42/p44 MAP kinases, mediated the induction of myocardin proteins by stretch in VSMCs. The conditioned medium from stretched VSMCs could induce the same increase in myocardin protein expression in non-stretched VSMCs (Figure 2A and B). These findings suggest that cyclic stretch regulated myocardin protein expression in VSMCs possibly via autocrine or paracrine mechanisms. 


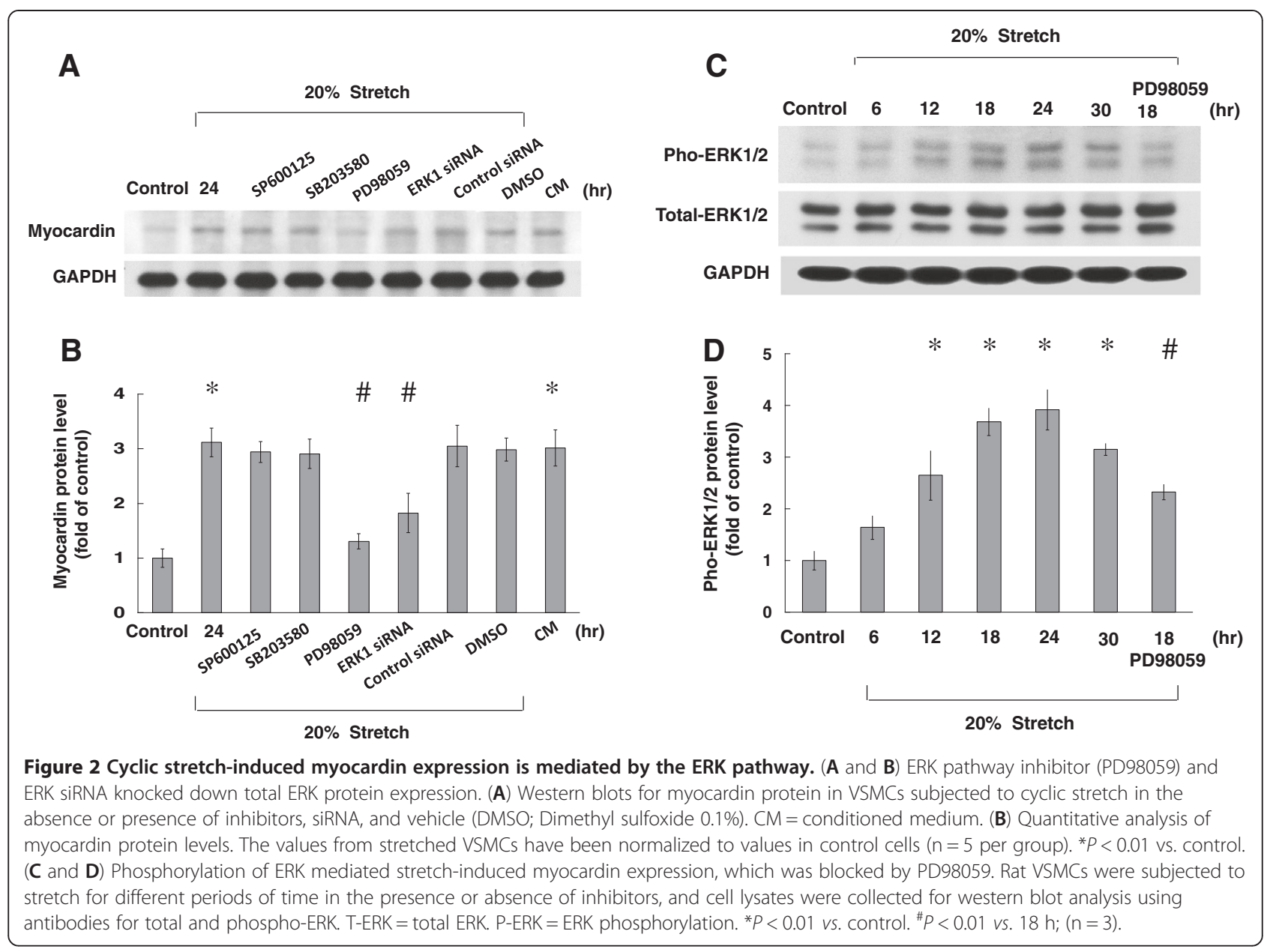

Cyclic stretch increases the phosphorylation of ERK protein in rat vascular smooth muscle cells

We also found that ERK protein phosphorylation increased to its maximal level $24 \mathrm{~h}$ after $20 \%$ cyclic stretch and declined gradually. The ERK pathway inhibitor (PD98059) could effectively block the phosphorylation of ERK protein (Figure 2C and D).

\section{Cyclic stretch stimulates secretion of angiotensin II from vascular smooth muscle cells}

As shown in Figure 3A, cyclic stretch significantly began to increase the AngII secretion from VSMCs and reached a peak at $6 \mathrm{~h}$ after stretch. Later, AngII levels declined gradually until $30 \mathrm{~h}$ (Figure 3A). Myocardin protein expression induced by cyclic stretch could effectively suppressed by AngII antibodies (Figure 3B and C). These results indicated that stretch causes VSMCs to secrete AngII.

\section{Exogenous addition of angiotensin II increases myocardin} protein expression

To investigate the direct effect of AngII on myocardin expression in VSMCs, AngII at different concentrations was administered to the cultured medium for $24 \mathrm{~h}$. As shown in Figure $3 \mathrm{~B}$ and $\mathrm{C}$, the effect of AngII on myocardin protein expression was dose-dependent. These findings suggested that exogenous addition of AngII also enhances myocardin expression without cyclic stretch. Addition of losartan $30 \mathrm{~min}$ before stretch significantly blocked the expression of myocardin induced by cyclic stretch for $24 \mathrm{~h}$.

\section{Cyclic stretch increases myocardin binding activity}

Cyclic stretch of VSMCs for 6-18 h significantly increased the DNA-protein binding activity of myocardin to SRF (Figure 4A). An excess of unlabelled myocardin oligonucleotide competed with the probe for binding myocardin protein, whereas an oligonucleotide containing a 2 bp substitution in the myocardin binding site did not compete for binding. Addition of ARB (losartan) and ERK pathway inhibitor (PD98059) 30 min before stretch abolished the DNA-protein binding activity induced by cyclic stretch. Exogenous administration of AngII to the VSMCs without stretch also increased myocardin-DNA binding activity (Figure 4A). These results demonstrated that stretch enhanced myocardin binding activity in VSMCs. 


\section{A}

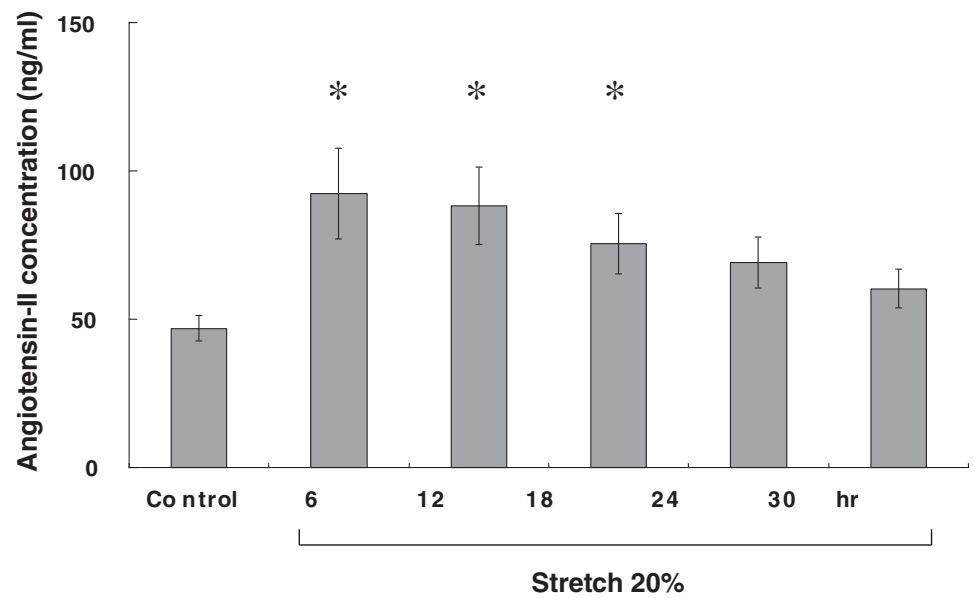

B

$20 \%$ Stretch, $24 \mathrm{hr}$ Ang2 (nM), $24 \mathrm{hr}$

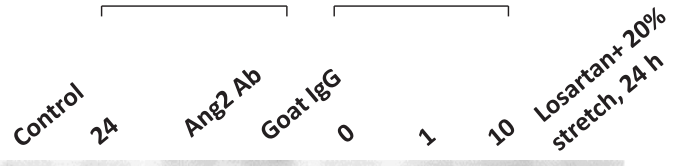

Myocardin

GAPDH

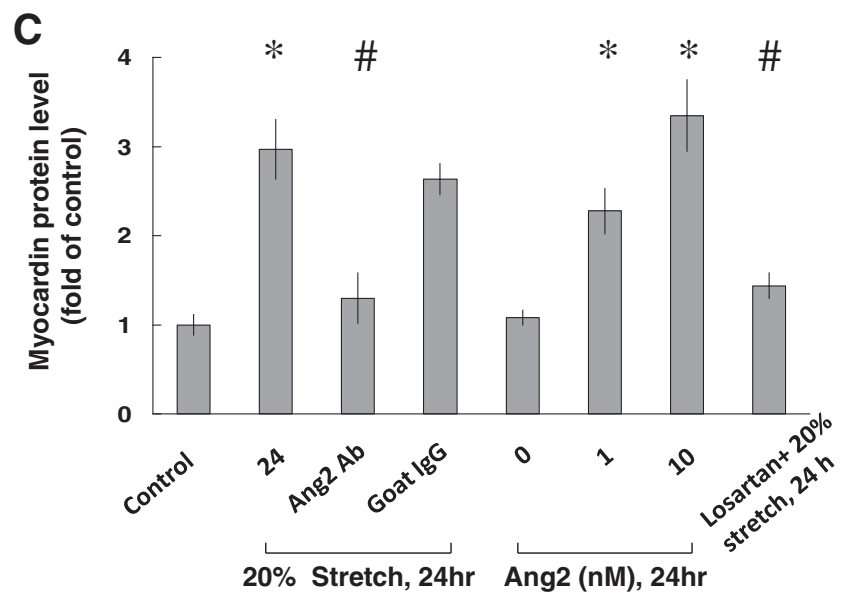

Figure 3 Angll mediates stretch-induced myocardin expression in rat VSMCs. (A) Angll was measured in the culture medium by a quantitative, competitive ELISA, using a specific anti-Angll antibody. Angll levels increased significantly $6 \mathrm{~h}$ after $20 \%$ stretch. (B and C) Either 20\% stretch or exogenous addition of Angll increased myocardin expression, and was inhibited by Angll antibodies or ARB (losartan). In this experiment, Western blots for myocardin protein in VSMCs subjected to cyclic stretch or exogenous addition of Angll in the absence or presence of inhibitors (Angll Ab or losartan) were performed. GAPDH was shown for equal amounts of protein loading in each lane. ${ }^{*} P<0.01 \mathrm{vs}$. control. ${ }^{\#} P<0.01$ vs. $24 \mathrm{~h}$ after stretch or $10 \mathrm{~h}$ after exogenous addition of Angll $(n=3)$.

\section{Cyclic stretch increases myocardin promoter activity}

\section{through the ERK pathway}

To study whether the myocardin expression induced by stretch is regulated at the transcriptional level, we cloned the promoter region of rat myocardin $(-968$ to +44$)$ and constructed a luciferase reporter plasmid (pGL3-Luc).
The myocardin promoter construct contains myocardin binding sites. As shown in Figure $4 \mathrm{~B}$ and $\mathrm{C}$, transient transfection experiments on VSMCs using this reporter gene revealed that stretch for $6 \mathrm{~h}$ significantly induced myocardin promoter activity. This result indicated that myocardin expression in VSMCs is induced at 


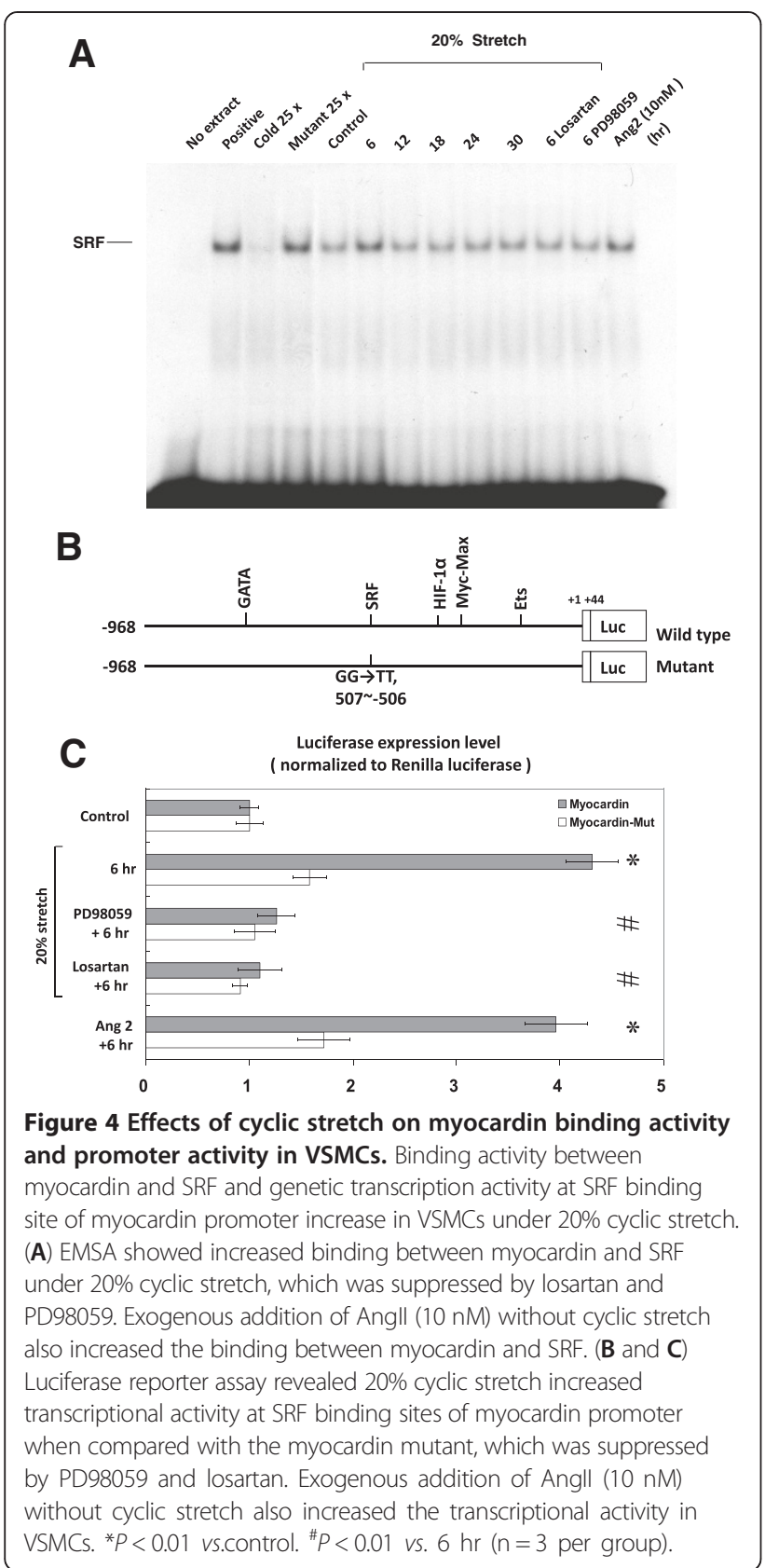

transcriptional level during cyclic stretch. When the myocardin binding sites were mutated, the increased promoter activity induced by stretch was abolished. Moreover, addition of ERK pathway inhibitor (PD98059) and ARB (losartan) caused an inhibition of transcription. These results suggested that the binding site in the myocardin promoter is essential for transcriptional regulation by cyclic stretch. In addition, we also found that exogenous addition of AngII without stretch increases the transcriptional activity in VSMCs (Figure 4C).

\section{Myocardin increases the migration of VSMCs}

Rat VSMCs cultured in the conditioned medium generated from stretched cells migrated significantly through the filter membrane compared with those cultured in non-conditioned medium (Figure 5A and B). Inhibition of myocardin activity was observed by ERK pathway inhibitor (PD98059), ARB (losartan), and myocardin siRNA, which decreased VSMC migration activity (Figure 5A and B). The migration activity of VSMCs was similar in controls and scrambled siRNA-treated groups. These findings suggest that myocardin mediates the migration of VSMCs induced by cyclic stretch.

\section{Cyclic stretch induces protein synthesis in VSMCs and VSMC hypertrophy}

We evaluated protein synthesis in VSMCs by measuring $\left[{ }^{3} \mathrm{H}\right]$ proline incorporation into the cells. Result showed increased protein synthesis in conditioned medium (CM) after cyclic stretch for 12 to $24 \mathrm{hr}$, which represented hypertrophic change of VSMCs (Figure 6). Pretreatment with ERK pathway inhibitor (PD98059), losartan, AngII antibody, and myocardin siRNA inhibited the protein synthesis induced by cyclic stretch. Exogenous addition of AngII also increased proline incorporation similar to the effect of cyclic stretch. We also used confocal microscopy to identify the effect of VSMC hypertrophy under cyclic stretch (Figure 7A and B). Confocal microscopy showed increased VSMC size $24 \mathrm{hr}$ after cyclic stretch, which represented a hypertrophic change of VSMCs (Figure 7B).

\section{In vivo aorta-caval shunt increases aortic myocardin protein expression}

Aorta-caval shunt was performed to explore whether myocardin expression increased under volume-overload in vivo. As shown in Figure 8, the myocardin protein expression in rat aorta significantly increased at days 1 to 3 after performing of aorta-caval shunt. It reached a maximum of 3.0-fold over the sham-operated rat and remained elevated up to 7 days. In addition, treatment with losartan $(10 \mathrm{mg} / \mathrm{kg} /$ day; from day 1 to day 5$)$ after performing aorta-caval shunt signigicantly suppressed myocardin protein expression (Figure $8 \mathrm{C}$ and $\mathrm{D}$ ). Confocal microcopy also revealed VSMC hypertrophy after creation of aorta-caval shunt for 3 days (Figure 7D).

\section{Discussion}

In this study, we demonstrated several significant or novel findings. Firstly, cyclic stretch upregulates myocardin expression in rat VSMCs; secondly, cyclic stretch induces AngII expression in VSMCs; thirdly, AngII acts as an autocrine factor to mediate the increased myocardin expression induced by cyclic stretch; fourthly, ERK MAP kinase and SRF transcriptional factor are involved in the signaling pathway of myocardin induction; and fifthly, 
A
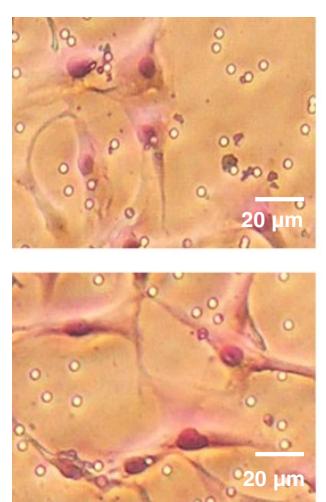

Stretch $24 \mathrm{hr}$

+ Losartan
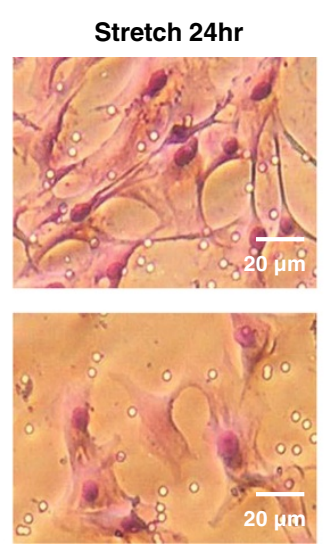

Stretch 24hr

+Myocardin siRNA
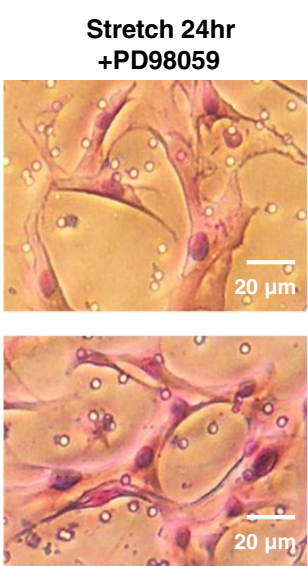

Stretch 24hr

+Control siRNA

\section{B}

\# \#

\#

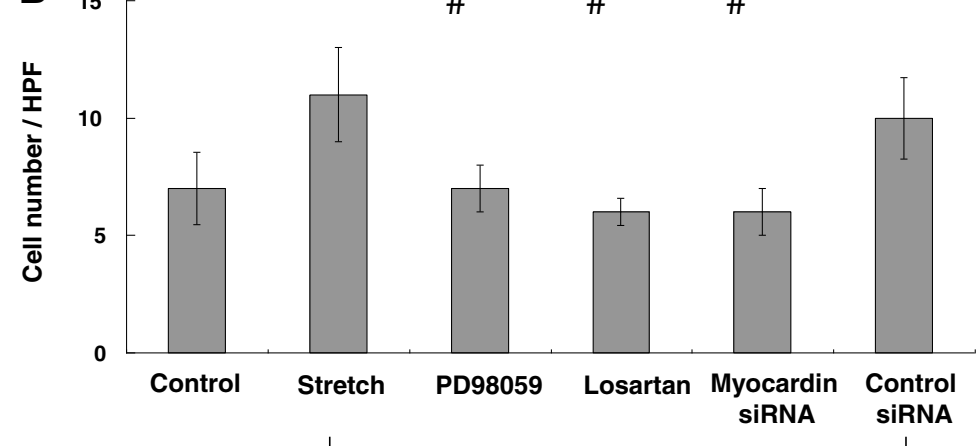

Stretch 20\%, 24hr

Figure 5 Effect of stretch on the migration of myocardin in VSMCs. (A) Condition medium (CM) was obtained from VSMCs after cyclic stretch for $24 \mathrm{~h}$. VSMCs migrating through the filter were stained. (B) The stained VSMCs were counted in four fields under a $400 \mathrm{X}$ high-power field (HPF). ${ }^{*} P<0.01$ vs. control. ${ }^{\#} P<0.05$ vs. $24 \mathrm{~h}(\mathrm{n}=3)$.

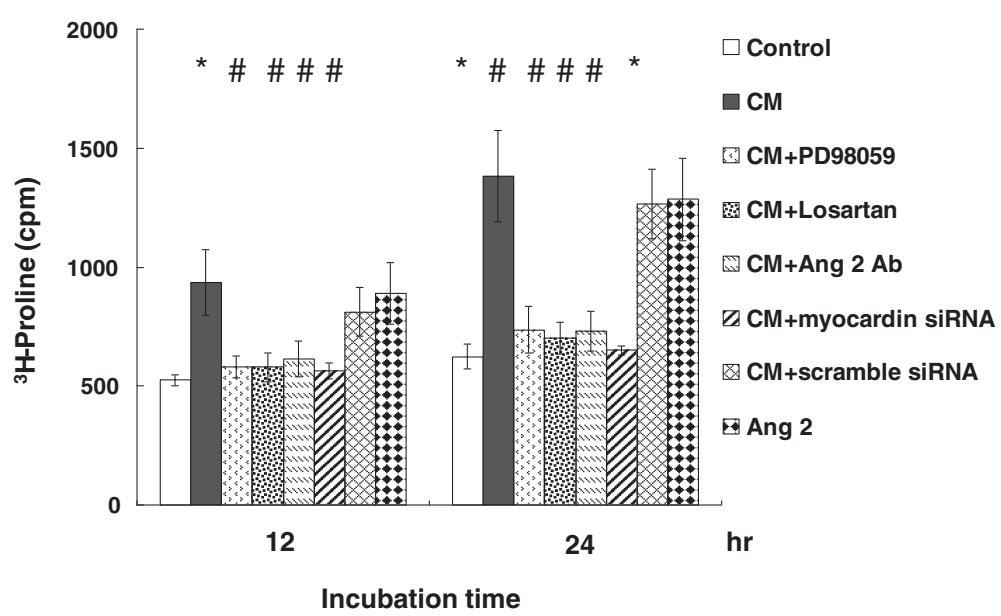

Figure 6 Protein synthesis in VSMCs increases after cyclic stretch. Incorporation of ${ }^{3} \mathrm{H}$-proline into VSMCs in condition medium (CM) increased after 20\% cyclic stretch and exogenous addition of Angll for 12 to 24 hrs and was suppressed by PD98059, losartan, Angll antibody, and myocardin siRNA. ${ }^{*} P<0.05$ vs. control. ${ }^{\#} P<0.05$ vs. cyclic stretch ( $n=6$ per group). 


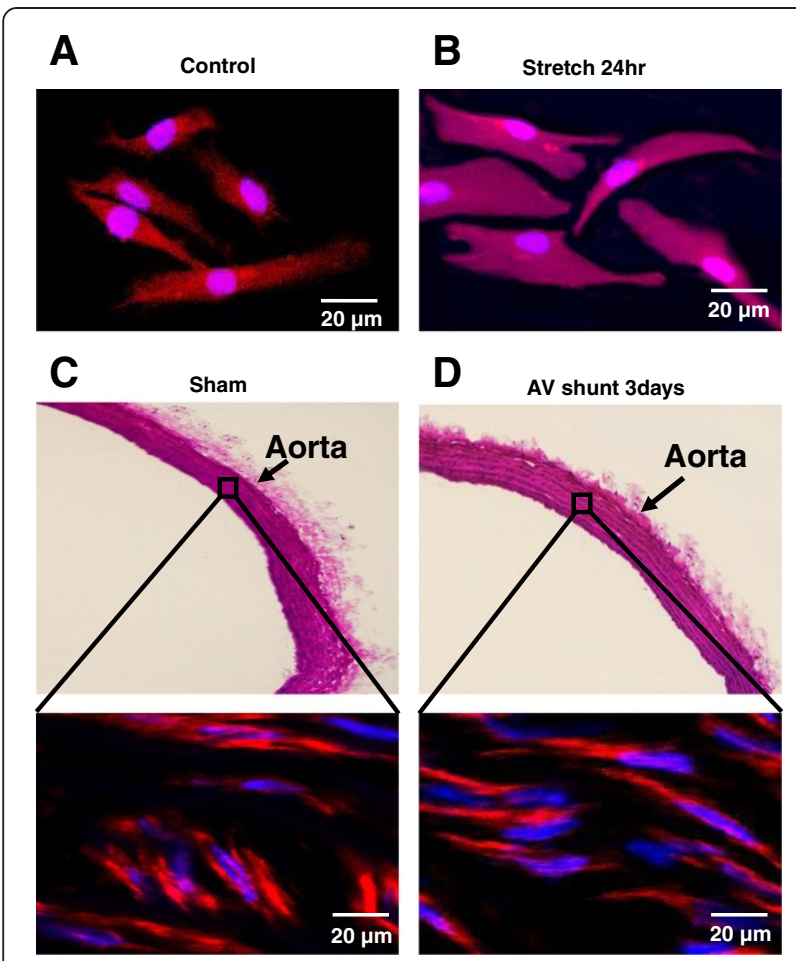

Figure 7 Cyclic stretch and aorta-caval shunt induce VSMC hypertrophy. Confocal microscopy showed increased VSMC size $24 \mathrm{hr}$ after cyclic stretch, which represented a hypertrophic change of VSMCs (Figure 7B). In addition, VSMC hypertrophy was also noted after creation of aorta-caval shunt for 3 days (Figure 7D).

in vivo acute hemodynamic overload increases aortic myocardin expression. Myocardin was upregulated in both a time- and load- dependent manner by cyclic stretch. Cyclic stretch of VSMCs increased both myocardin protein and mRNA expression.

In our study, exogenous addition of AngII to nonstretched VSMCs was also sufficient to induce similar myocardin protein expression as that observed in stretched VSMCs. These results provide the first evidence that AngII mediates cyclic stretch-induced expression of myocardin in VSMCs. Our study revealed that AngII acts as an autocrine mediator in response to cyclic stretch in VSMCs. Previously, another study identified that AngII enhanced myocardin expression through AngII type 1 (AT1) receptor results in VSMC hypertrophy [12]. We also have previously demonstrated that hypoxia in cardiomyocytes increased AngII secretion and myocardin expression and finally resulted in cardiac myocyte hypertrophy through the ERK pathway [13]. In this study, we found that cyclic stretch also enhanced myocardin expression by AngII secretion and the ERK pathway, which had not been identified by previous studies.

However, one study found no increased concentration of AngII in the medium collected from porcine VSMCs at 24 and $48 \mathrm{~h}$ after $25 \%$ stretch [36]. Sotoudeh et al. used pulmonary VSMCs, whereas our study used rat aortic VSMCs. Different species, stretch intension, and stretch time may explain the discrepancy. Our results suggest that AngII is responsible for myocardin-DNA binding in VSMCs. In this study, we demonstrated that cyclic stretch stimulation of myocardin-DNA binding activity required at least phosphorylation of ERK since ERK pathway inhibitor (PD98059) and ERK siRNA abolished the myocardin/SRF binding activity. PD98059, a potent and specific inhibitor of ERK MAP kinase, also inhibited the myocardin expression induced by stretch, whereas inhibitors of $\mathrm{p} 42 / \mathrm{p} 44, \mathrm{p} 38$, and c-JUN MAP kinase did not have this inhibitory effect. Thus, ERK MAP kinase is an important intracellular signaling pathway that regulates myocardin expression. We also demonstrated that ERK siRNA significantly inhibited myocardin expression induced by stretch. ARB likewise had an inhibitory effect on the stretch-induced myocardin expression. Since ARB is an AngII inhibitor, and mechanical stretch is known to affect the production of AngII, [37], our findings potentially indicate that AngII has a role in the induction of myocardin by mechanical stretch. In this study, we demonstrated via promoter activity assay that increased transcriptional activity of myocardin promoter by cyclic stretch was SRF dependent. These data imply that the ERK MAP kinase pathway, but not the other MAP kinase pathway, is the major pathway involved in the induction of myocardin by stretch and that it mediates the increased binding activity of myocardin and transcription to VSMCs.

Mechanical stretch can modulate several different cellular functions in VSMCs. These functions include cell alignment and differentiation, migration, survival or apoptosis, vascular remodeling, and autocrine or paracrine functions [37]. However, use of different kinds of VSMCs (venous or arterial) and various species of animals used in different studies (mouse, rat, rabbit, swine and others), have resulted in sometimes controversial findings [37]. Most of these studies used in vitro models. However, the cellular functions induced by in vitro mechanical stretch may not accurately represent cellular function in vivo [37]. So, more studies are necessary to identify the real effects of mechanical stretch on VSMC functions and the mechanisms by which they occurr. Our study further confirmed the increased aortic myocardin expression in acute hemodynamic overload as that occurring with aorta-caval shunts. It has been previously reported that myocardin protein expression increased in the carotid artery balloon injury model in rats [11], suggesting myocardin may be enhanced during acute hemodynamic overload in vivo. The increased myocardin protein expression following acute hemodynamic overload may contribute to the regulation of 


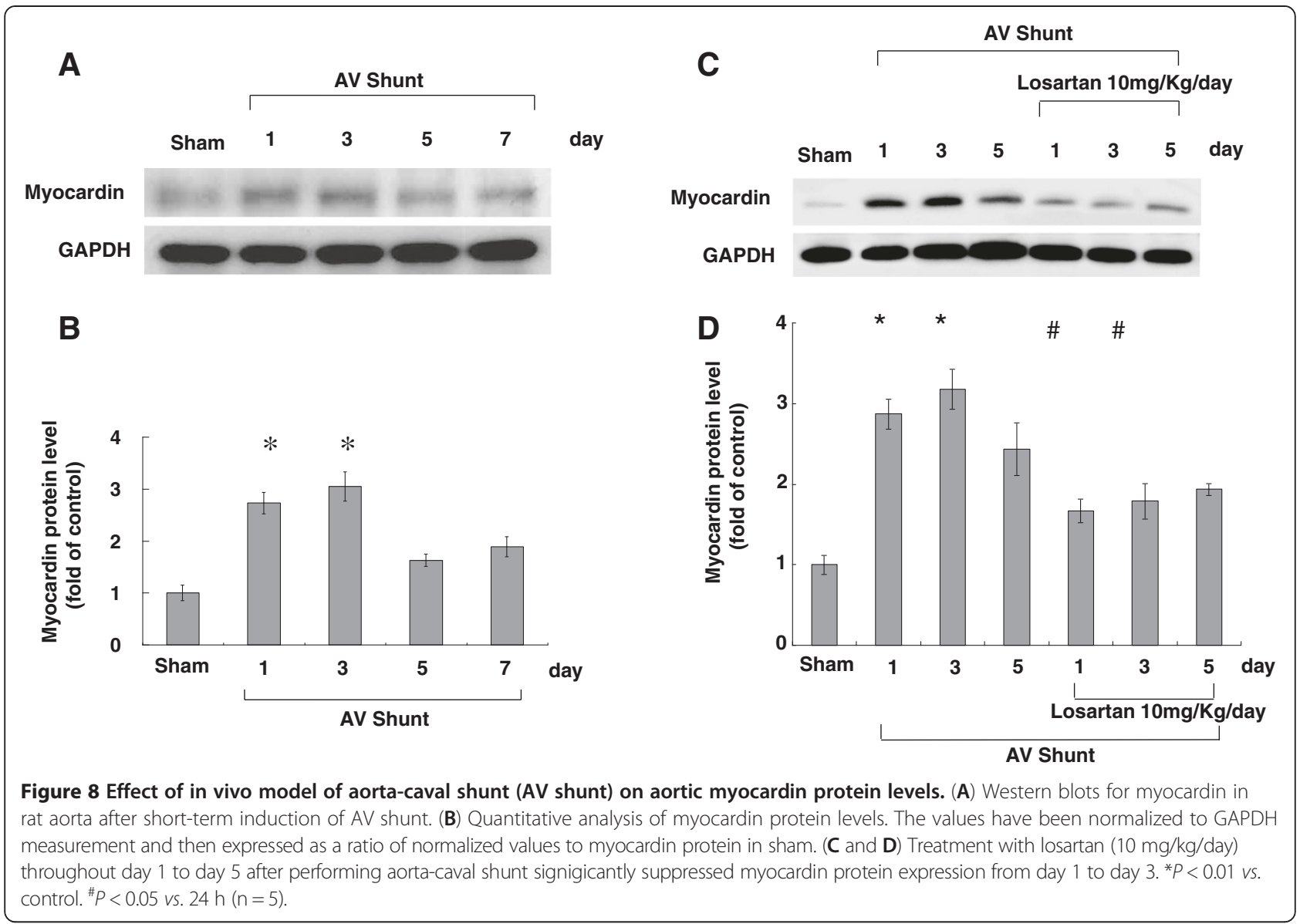

vascular repair and remodeling, which involves VSMC proliferation [17].

With regard to the clinical application of cyclic stretch on VSMCs, mechanical stretch activates multiple intracellular signaling networks and regulates gene expressions and functional responses in VSMCs. The cellular and molecular effects of mechanical stretch on vascular cells may provide new insights in the pathogenesis of vascular diseases and therapeutic potentials. Mechanical stretch can modulate several different cellular functions in VSMCs, including cell alignment and differentiation, migration, survival or apoptosis, vascular remodeling, and autocrine and paracrine functions. Arterial VSMCs are aligned primarily in the circumferential direction in the media of the artery. Mechanical stretch from pulsatile blood flow is one of the key factors in regulating vascular remodeling. VSMC migration is important in the development of vascular diseases, including atherosclerosis and post-angioplasty restenosis. VSMC migration is found more frequently in curved and bifurcating blood vessels, which are exposed to non-laminar blood flow, than in straight arterial segments exposed to laminar blood flow. In this, we have also demonstrated that mechanical stretch increased the migration of VSMCs. The gene expression induced by mechanical stretch may be relevant to pathological complications in the cardiovascular system, including atherosclerosis, plaque instability and hypertension. The induction of genes by mechanical stretch may play a role in vascular remodeling. Understanding the molecular mechanisms regulating VSMC remodeling, migration, and proliferation under mechanical stretch supports the clinical application of ACEI (angiotensin-converting enzyme inhibitors), ARB, and statin in cardiac protection and in the prevention of vascular diseases. Therefore knowledge of the impact of mechanical stretch on VSMCs is vital in the understanding of the pathogenesis of cardiovascular diseases and is crucial in providing new insights into the prevention and therapy of cardiovascular diseases.

\section{Conclusion}

In summary, our study reports for the first time that cyclic mechanical stretch enhances myocardin expression in cultured rat VSMCs. The stretch-induced myocardin is mediated by AngII through the ERK pathway. 


\section{Abbreviations}

VSMCs: Vascular smooth muscle cells; SRF: Serum response factor; siRNA: Small interfering RNA; Angll: Angiotensin II; mAbs: Monoclonal antibodies; JNK: C-Jun N-terminal kinase; MAPK: Mitogen-activated protein kinase; ERK: Extracellular signal-regulated kinase; ARB: Angll type 1 receptor blockers; RT: Reverse transcription; PCR: Polymerase chain reaction;

ELISA: Enzyme-linked immunosorbent assay; EMSA: Electrophoretic motility shift assay; DMEM: Dulbecco's modified Eagle's/F12 medium;

CM: Conditioned medium; AT1: Receptor: Angll type 1 receptor.

\section{Competing interests}

The authors declare that they have no competing interests.

\section{Authors' contributions}

CZC participated in the design of the study and drafted the paper. BWW made substantial contributions to conception and design, acquisition of data or analysis, and interpretation of data. KGS participated in the design of the study and final approval of the paper prior to submission. All authors read and approved the final manuscript.

\section{Acknowledgement}

This study was sponsored in part by Shin Kong Wu Ho-Su Memorial Hospital, Taipei, Taiwan.

\section{Author details}

${ }^{1}$ School of Medicine, Fu-Jen Catholic University, New Taipei, Taiwan. ${ }^{2}$ Division of Cardiology, Shin Kong Wu Ho-Su Memorial Hospital, 95 Wen-Chang Rd, Taipei, Taiwan. ${ }^{3}$ Graduate Institute of Clinical Medicine, College of Medicine, Taipei Medical University, Taipei, Taiwan.

Received: 9 April 2013 Accepted: 10 July 2013 Published: 15 July 2013

\section{References}

1. Gunningham KS, Gotlieb Al: The role of shear stress in the pathogenesis of atherosclerosis. Lab Invest 2005, 85(1):9-23.

2. Kakisis JD, Liapis CD, Sumpio BE: Effects of cyclic strain on vascular cells. Endothelium 2004, 11(1):17-28.

3. Li C, Xu Q: Mechanical stress-initiated signal transduction in vascular smooth muscle cells in vitro and in vivo. Cell Signaling 2007, 19(5):881-891.

4. Lehoux S, Esposito B, Merval R, Loufrani L, Tedgui A: Pulsatile stretch-induced extracellular signal-regulated kinase $1 / 2$ activation in organ culture of rabbit aorta involves reactive oxygen species. Arterioscler Thromb Vasc Biol 2000, 20(11):2366-2372.

5. Albinsson S, Hellstrand P: Integration of signal pathways for stretch-dependent growth and differentiation in vascular smooth muscle cell. Am J Physiol Cell Physiol 2007, 293(2):C772-C782.

6. Wang D, Chang PS, Wang Z, Sutherland L, Richardson JA, Small E, Krieg PA, Olson EA: Activation of cardiac gene expression by myocardin, a transcriptional cofactor for serum response factor. Cell 2001, 105(7):851-862.

7. Parlakian A, Tuil D, Hamard G, Tavernier G, Hentzen D, Concordet JP, Paulin D, Li Z, Daegelen D: Targeted inactivation of serum response factor in the developing heart results in myocardial defects and embryonic lethality. Mol Cell Biol 2004, 24(12):5281-5289.

8. Teg Pipes GC, Creemers EE, Olson EN: The myocardin family of transcriptional coactivators: versatile regulators of cell growth, migration, and myogenesis. Genes Dev 2006, 20(12):1545-1556.

9. Milano JM: Channeling to myocardin. Circ Res 2004, 95(4):340-342.

10. Torrado M, Lopez E, Centeno A, Medrano C, Castro-Beiras A, Mikhailov AT: Myocardin mRNA is augmented in the failing myocardium: expression profiling in the porcine model and human dilated cardiomyopathy. J Mol Med 2003, 81(9):566-577.

11. Liu ZP, Wang Z, Yanagisawa H, Olson EN: Phenotypic modulation of smooth muscle cells through interaction of Foxo4 and myocardin. Dev Cell 2005, 9(2):261-270.

12. Yoshida T, Hoofnagle MH, Owens GK: Myocardin and Prx1 contribute to angiotensin II-induced expression of smooth muscle a-actin. Circ Res 2004, 94(8):1075-1082.
13. Chiu CZ, Wang BW, Chung TH, Shyu KG: Angiotensin II and ERK pathway mediate the induction of myocardin by hypoxia in cultured rat neonatal cardiomyocytes. Clin Sci 2010, 119(7):273-282

14. Chiu CZ, Wang BW, Shyu KG: Use of atorvastatin to inhibit hypoxia-induced myocardin expression. Eur J Clin Invest 2012, 42(5):564-571.

15. Chiu CZ, Wang BW, Shyu KG: Atorvastatin, valsartan, and n-acetylcysteine prevent cardiac hypertrophy and overexpression of myocardin in pressure- overloaded rat heart. Acta Cardiologica Sinica 2012, 28(4):286-298.

16. Riha GM, Lin PH, Lumsden AB, Yao Q, Chen C: Roles of hemodynamic forces in vascular cell differentiation. Ann Biomed Eng 2005, 33(6):772-779.

17. Chang H, Shyu KG, Wang BW, Kuan P: Regulation of hypoxia inducible factor 1-a by cyclical mechanical stretch in rat vascular smooth muscle cells. Clin Sci 2003, 105(4):447-456.

18. Haga JH, Li YJ, Chien S: Molecular basis of the effects of mechanical stretch on vascular smooth muscle cells. J Biomech 2007, 40(10):947-960.

19. Richard MN, Deniset JF, Kneesh AL, Blackwood D, Pierce GN: Mechanical stretching stimulates smooth muscle cell growth, nuclear protein import, and nuclear pore expression through mitogen-activated protein kinase activation. J Bio/Chem 2007, 282(32):23081-23088.

20. Shyu KG, Chang ML, Wang BW, Kuan P, Chang H: Cyclical mechanical stretching increases the expression of vascular endothelial growth factor in rat vascular smooth muscle cells. J Formos Med Assoc 2001, 100(11):741-747.

21. Hellstrand P, Albinson S: Stretch-dependent growth and differentiation in vascular smooth muscle: role of the actin cytoskeleton. Can J Physiol Pharmacol 2005, 83(10):869-875.

22. Morrow D, Sweeney C, Birney YA, Cummins PM, Walls D, Redmond EM, Cahill PA: Cyclic strain inhibits notch receptor signaling in vascular smooth muscle cells in vitro. Circ Res 2005, 96(5):567-575.

23. Lehoux S, Castier Y, Tedgui A: Molecular mechanism of the vascular response to haemodynamic forces. J Int Med 2006, 259(4):381-392.

24. Li YS, Haga JH, Chien S: Molecular basis of the effects of shear stress on vascular endothelial cells. J Biomech 2005, 38(10):1949-1971.

25. Taber $L A$ : A model for aortic growth based on fluid shear and fiber stresses. J Biomech Eng 1998, 120(3):348-354.

26. Liu SQ: Focal expression of angiotensin II type 1 receptor and smooth muscle cell proliferation in the neointima of experimental vein grafts: relation to eddy blood flow. Arterioscler Thromb Vasc Biol 1999, 19(11):2630-2639.

27. Wasserman SM, Mehraban F, Komuves LG: Gene expression profile of human endothelial cells exposed to sustained fluid shear stress. Physiol Genomics 2002, 12(1):13-23.

28. Andersson M, Karisson L, Svensson PA: Differential global gene expression response patterns of human endothelium exposed to shear stress and intraluminal pressure. J Vasc Res 2005, 42(5):441-452.

29. Cheng WP, Wang BW, Chen SC, Chang H, Shyu KG: Mechanical stretch induces the apoptosis regulator PUMA in vascular smooth muscle cells. Cardiovasc Res 2012, 93(1):181-189.

30. Wang BW, Chang H, Shyu KG: Regulation of resistin by cyclic mechanical stretch in cultured rat vascular smooth muscle cells. Clin Sci 2009, 118(3):221-230.

31. Stavri GT, Zachary IC, Baskerville PA, Martin JF, Erusalimsky JD: Basic fibroblast growth factor upregulates the expression of vascular endothelial growth factor in vascular smooth muscle cells: synergistic interaction with hypoxia. Circulation 1995, 92(1):1-14.

32. Shyu KG, Chao YM, Wang BW, Kuan P: Regulation of discoidin domain receptor 2 by cyclic mechanical stretch in cultured rat vascular smooth muscle cells. Hypertension 2005, 46(3):614-621.

33. Cheng JJ, Wung BS, Chao YJ, Wang DL: Cyclical strain enhances adhesion monocytes to endothelial cells by increasing intercellular adhesion molecular-1 expression. Hypertension 1996, 28(3):386-391.

34. Shyu KG, Ko WH, Yang WS, Wang BW, Kuan P: Insulin-like growth factor-1 mediates stretch-induced upregulation of myostatin expression in neonatal rat cardiomyocytes. Cardiovasc Res 2005, 68(3):405-414

35. Liang YJ, Lai LP, Wang BW, Juang SJ, Chang CM, Leu JG: Shyu KG: Mechanical stretch enhances serotonin $2 \mathrm{~B}$ receptor modulating brain natriuretic peptide through nuclear factor-kappaB in cardiomyocytes. Cardiovasc Res 2006, 72(2):303-312. 
36. Sotoudeh M, Li YS, Yajima N, Chang CC, Tsou TC, Wang Y, Usami S, Ratcliffe A, Chien S, Shyy JY: Induction of apoptosis in vascular smooth muscle cells by mechanical stretch. Am J Physiol Heart Circ Physiol 2002, 282(5):H1709-716

37. Shyu KG: Cellular and molecular effects of mechanical stretch on vascular cells and cardiac myocytes. Clin Sci 2009, 116(5):377-389.

doi:10.1186/1423-0127-20-50

Cite this article as: Chiu et al: Effects of cyclic stretch on the molecular regulation of myocardin in rat aortic vascular smooth muscle cells. Journal of Biomedical Science 2013 20:50.

\section{Submit your next manuscript to BioMed Central and take full advantage of:}

- Convenient online submission

- Thorough peer review

- No space constraints or color figure charges

- Immediate publication on acceptance

- Inclusion in PubMed, CAS, Scopus and Google Scholar

- Research which is freely available for redistribution 